\title{
STATISTICAL CHARACTERIZATION OF AEROSOLS' LIQUID WATER CONTENT AND VISIBILITY FOR TERRESTRIAL FSO LINKS
}

Liquid water content (LWC) is a microphysical parameter which is used to characterize fog/cloud and other particulates. In the absence of precipitation higher amount of LWC depicts dense fog, significantly reducing visibility and causing performance degradation of free-space optical (FSO) links for a non-negligible amount of time. A measurement campaign in Prague (Czech Republic) has recorded the specific attenuation caused by fog in terrestrial FSO links along with visibility, liquid water content (LWC) and integrated particle surface area (PSA) for operational wave-lengths of $1550 \mathrm{~nm}$ and $830 \mathrm{~nm}$ (installed at two different path links). This contribution presents and analyzes 5 months real time measured data of LWC and visibility for terrestrial FSO links in an attempt to find the best fit distribution model for aerosol's liquid water content and visibility for terrestrial FSO links under reduced visibility conditions. The Probability Density Function (PDF) estimation of visibility and LWC puts forth the Gamma distribution as the best fit distribution for both. The results provide the statistical behaviour of LWC and visibility, which is of high importance for Optical Wireless systems and networks.

Keywords: Free-space Optics (FSO), Optical Wireless Communication, Liquid Water Content (LWC), Visibility.

\section{Introduction}

Free-space optical (FSO) links are supposed to operate through atmosphere which contains fog, smoke, dust, rain, smog and charged particles. The terrestrial FSO link provide a viable last mile solution for high speed connectivity without the need of digging necessary to lay the conventional fiber [1]. Atmospheric particles like fog, snow, etc attenuate the transmitted signal propagating through the line-of-sight FSO links. Among all the different attenuating factors, fog is the most serious deterrent [2,3]. Fog can be characterized by liquid water content (LWC), optical visibility, drop size distribution and temperature [4]. The scattering, absorption and extinction of laser beam propagating through the atmosphere are associated with fog droplets sizes, their effective radii [3] and the microphysical properties of fog, specifically liquid water content $[3,5]$.

The LWC is the measure of total mass of water per unit volume of the droplets in air. The LWC is expressed in $\mathrm{g} / \mathrm{m}^{3}$. LWC is used to characterize the different types of fog/clouds. In the absence of precipitation higher amount of liquid water content decreases visibility and indicates dense fog $[5,6]$.
Fog is an accumulation of water droplets or ice crystal fines accompanied by hygroscopic, water-saturated fine particles that reduce visibility. Under dense fog conditions the visibility decreases below $100 \mathrm{~m}$ and cause sever signal attenuation for FSO links [2]. For light fog the visibility remains in the range of 500-1000 m relatively less deterrent for FSO links.

For wide spread acceptability of FSO links there is need to investigate thoroughly different weather impairment on FSO links [7]. The prediction of optical attenuation in lower atmospheric visibility ranges due to water hazes, fogs, and clouds has been thoroughly investigated and researched [8].

To the best of the authors' knowledge this is 1st attempt to find a distribution model for LWC for terrestrial FSO links. Fog drop size distribution is widely modelled as a modified gamma distribution $[9,10]$. The current work found Gamma distribution as a best fit model for visibility from 5 months measured data. Curve fitting techniques have been used to find the best fit PDF for LWC and visibility. All the continuous distributions have been compared using the Quantile-Quantile Plot (Q-Q plot). Here we presented the two best fit distributions for the analysis. The current work is

\footnotetext{
* Muhammad Saeed Khan ${ }^{1,5}$, Roberto Nebuloni ${ }^{2}$, Martin Grabner ${ }^{3}$, Carlo Capsoni ${ }^{4}$, Erich Leitgeb ${ }^{1}$, Vaclav Kvicera ${ }^{3}$

${ }^{1}$ Institute of Microwave and Photonic Engineering, Graz University of Technology, Graz, Austria

${ }^{2}$ IEIIT-CNR (Italian National Research Council), Milan, Italy

${ }^{3}$ Department of Frequency Engineering, Czech Metrology Institute, Prague, Czech Republic

${ }^{4}$ Politecnico di Milano, Italy

${ }^{5}$ COMSATS Institute of Information Technology, Wah Cantt, Pakistan

E-mail: msaeedbaloch@gmail.com
} 
of high importance for optical wireless communication and networks and will provide a thorough understanding of visibility and LWC and their variations.

\section{Experimental Setup}

The measurement campaign was carried out at the Department of Frequency Engineering, Czech Metrology Institute (CMI) Prague, Czech Republic from 08. 01. 2009 till 31.05.2009. Prague is located at latitude $50^{\circ} 05^{\prime} 12^{\prime \prime} \mathrm{N}$ longitude $14^{\circ} 24^{\prime} 59^{\prime \prime} \mathrm{E}$ and at altitude of $191 \mathrm{~m}$. Prague has continental weather. Prague has the air mean temperature $10.4^{\circ} \mathrm{C}$ from 1971-2000 [11]. The FSO systems were installed 26 meters above the ground level. The link margin of the two FSO systems allows the measurement of specific attenuation up to $180 \mathrm{~dB} / \mathrm{km}$ for $830 \mathrm{~nm}$ wavelength systems and $130 \mathrm{~dB} / \mathrm{km}$ for $1550 \mathrm{~nm}$ system. Optical calibration was performed before deploying the FSO devices. A received power is obtained from the calibrated Received Signal Strength Indicator (RSSI) signal of the FSO link. Meteorological conditions are identified by means of a color video camera and an automatic weather observation system located near the FSO receivers. The system uses Vaisala sensors for the measurement of temperature, humidity, air pressure, velocity and direction of the wind. The VAISALA PWD 11 equipment measures the atmospheric visibility ( $5 \%$ definition) values in the range from $50 \mathrm{~m}$ up to $2000 \mathrm{~m}$ using forward scattered light in the angle of $45^{\circ}$. The PVM- 100 device is used to measure liquid water content $\mathrm{LWC}\left(\mathrm{g} / \mathrm{m}^{3}\right)$ and integrated particle surface area PSA $\left(\mathrm{cm}^{2} / \mathrm{m}^{3}\right)$ of fog.

The meteorological data is synchronized in time with the hydrometer attenuation measurement. The received FSO signal levels and the meteorological data are recorded synchronously on a PC's hard disk. In this article we analyzed 5 months real time measurement data of reduced visibility and LWC due to fog for terrestrial FSO links. In the measurement campaign, we sampled data at rate of one sample per minute. The other instruments were calibrated in a way to measure the specific quantity at the last second of the every minute in parallel with the optical attenuations. We selected data set for analysis where the visibility was less than $1 \mathrm{Km}$. Figs 1 and 5 shows the reduced data set of LWC and Visibility. It is important to note that the time axis of Figs 1 and 5 is not continuous.

\section{Results and Analysis}

The department of Frequency Engineering at Czech Metrology Institute is conducting measurement campaign at path links of $100 \mathrm{~m}$ and $853 \mathrm{~m}$ using the wavelengths of $1550 \mathrm{~nm}, 830 \mathrm{~nm}$ and $850 \mathrm{~nm}$ along with visibility, LWC and integrated particle surface area (PSA). A 5 months measured data of visibility and LWC were collected for detailed analysis. In the measurement campaign we sampled data at a rate of one sample per minute. The other instruments were calibrated in a way to measure the specific quantity at the last second of the every minute in parallel with the optical attenuation. In metrology theory, it is admitted that fog exists when vis- ibility is decreased to less to less than $1 \mathrm{~km}$. Therefore we selected the data set for analysis where the visibility was less than $1 \mathrm{~km}$. Fig. 1 shows the reduced data set. It is also important to remark that the time axis in the figure is not continuous. The detailed statistical analysis to find the best suited distribution model for visibility and LWC data is provided in subsequent sections.

\section{A. Visibility}

Visibility is defined as the distance to an object at which the image contrast drops to a certain percentage of the original contrast of the object, equivalent to a certain transmission threshold tTH over the atmospheric path. Two different definitions for threshold exist, $2 \%$ and $5 \%$. The $5 \%$ transmission threshold is more commonly used at airports to denote the "runway visibility range" RVR $[12,13]$. Another meteorological definition of visibility is the actual distance at which a person can discern an ideal dark object against the horizon sky [14]. The visibility range is technically measured at the center of sensitivity for the human eye (where the sensitivity of the human eye is maximum), at $550 \mathrm{~nm}$ with a spectral band width of typically $250 \mathrm{~nm}$. Different visibility based empirical models have been proposed to calculate optical attenuation from visibility, Kim [15], Pierce [16], Kruse [17] and Al-Naboulsi [18]. Here we presented the statistical characterization of 5 months measured visibility data. The analysis of measured visibility is provided in Fig. 1.

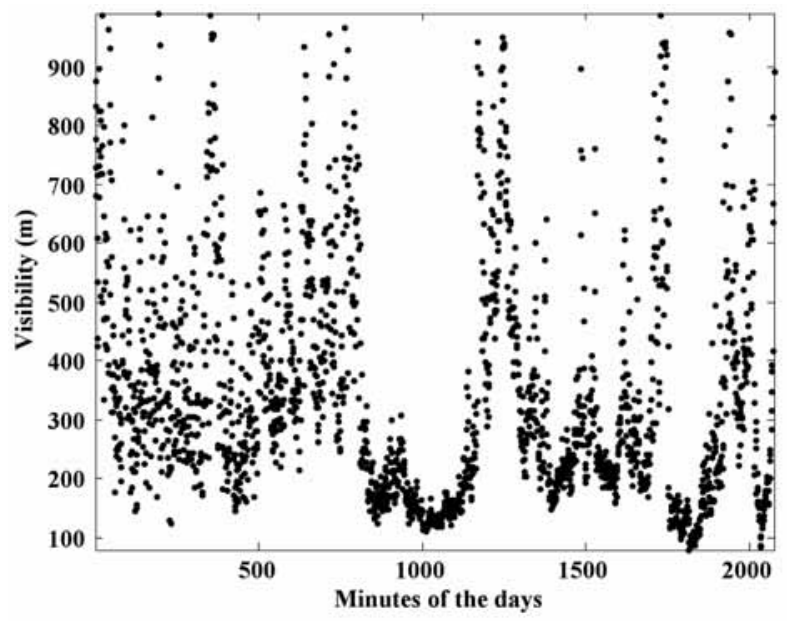

Fig. 1 Analysis of measured visibility

The horizontal axis of Fig. 1 shows the minutes of the days and the vertical axis shows the visibility measured in meters. It is clear from Fig. 1 that there occurred certain dense fog events when the visibility is less than $100 \mathrm{~m}$. The descriptive statistics of the visibility is provided in Table 1 .

It is important to mention that the minimum value of measured visibility is $80 \mathrm{~m}$ (from Table 1 ) while the maximum value is $991 \mathrm{~m}$. And also the skewness of the data is positive which shows that the right tail of the distribution will be longer as compared to the left tail. 
Descriptive statistics of the measured visibility

Tab. 1

\begin{tabular}{|c|c|c|}
\hline Serial No & Statistical Quantity & Values \\
\hline 1 & Sample Size & 2073 \\
\hline 2 & Range & 911 \\
\hline 3 & Mean & 343.74 \\
\hline 4 & Variance & 37688.0 \\
\hline 5 & Std. Deviation & 194.13 \\
\hline 6 & Std. Error & 4.2639 \\
\hline 7 & Skewness & 1.1366 \\
\hline 8 & Min & 80 \\
\hline 9 & $25 \%(Q 1)$ & 197.5 \\
\hline 10 & $50 \%($ Median) & 291 \\
\hline 11 & $75 \%(Q 3)$ & 443 \\
\hline 12 & $90 \%$ & 637.2 \\
\hline 13 & $95 \%$ & 752.2 \\
\hline 14 & Max & 991 \\
\hline
\end{tabular}

After having explored the descriptive statistics we proceed further to find the best fit distribution for visibility measured data. We compared all the continuous distribution on the visibility data by analysing the probability density function (PDF) and Cumulative distribution function (CDF). We performed comparison among all the distribution by comparing Quantile-Quantile-Plot (QQ plot). QQ plot is used for comparing two probability distributions by plotting their quantiles against each other. Here we show the results of two best fitted distribution for visibility data. The PDF and CDF of the measured visibility data is provided in Figs 2 and 3 respectively.

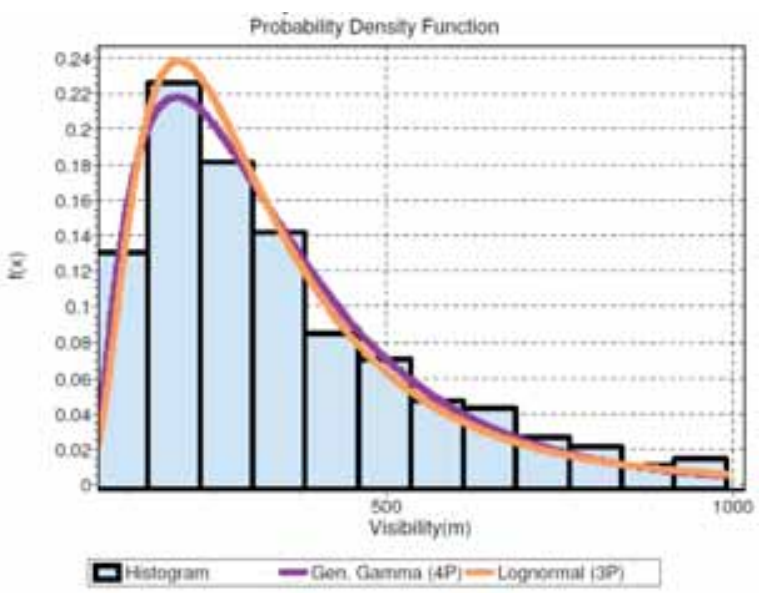

Fig. 2 PDF over the histogram of measured visibility data

Figs. 2 and 3 show that the selected distribution models can be used for statistical characterization of the behavior of measured visibility data. We performed the goodness of fit by observing their

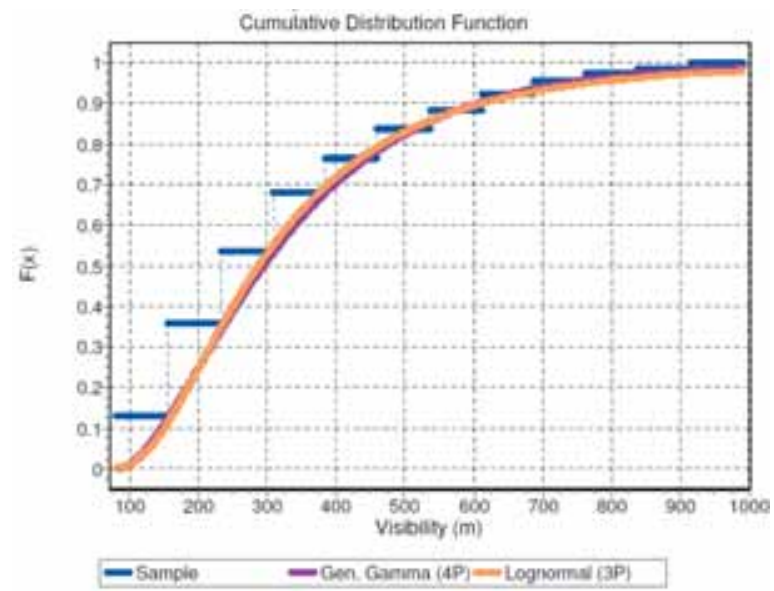

Fig. 3 CDF of the measured visibility

QQ plot of measured visibility to find the best fit model between the selected two models. The QQ plot of measured visibility is provided in Fig. 4. In Fig. 4 it is clear that Gamma distribution is performing well as compared to Lognormal distribution for measured visibility. The QQ plot for Gamma distribution is following the normal line as compared to that of Lognormal distribution.

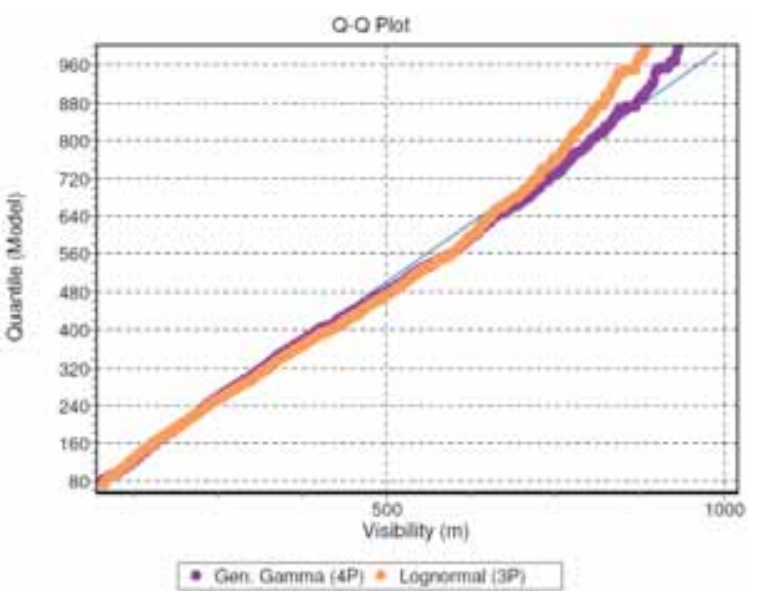

Fig. 4 QQ Plot for Visibility data

The probability density function for Gamma distribution is provided in equation (1).

$$
f(x)=\frac{\kappa(x-y)^{\kappa \alpha-1}}{\beta^{\kappa \alpha} \Gamma(a)} \exp (-((x-y) / \beta) \kappa)
$$

Where $\kappa$ is a continuous shape parameter $(\kappa>0)$, continuous shape parameter $(\alpha>0), \beta$ is scale parameter $(\beta>0)$ and $\gamma$ is location parameter. We computed the optimum parameters for Gamma distribution for measured visibility which are provided in Table 2 . 
Optimum parameters for best fitted distribution

Tab. 2

\begin{tabular}{|l|l|l|}
\hline Description & Distribution Model & Parameters \\
\hline Visibility & Gamma & $\begin{array}{l}\kappa=0.79766, \alpha=2.8018, \\
\beta=69.203, \gamma=78.538\end{array}$ \\
\hline LWC & Gen. Gamma & $\begin{array}{l}\gamma=0.96962, \alpha=1.4269, \\
\beta=0.0438\end{array}$ \\
\hline
\end{tabular}

We concluded that Gamma distribution (4 parameters Gamma Distribution model) is the best suited model for measured visibility in the absence of precipitation. The proposed distribution model can be used under fog conditions.

\section{B. Liquid water content}

LWC is the measurement of mass of water in fog/cloud in a specified amount of dry air. LWC can be expressed as $\mathrm{g} / \mathrm{m}^{3}$. LWC is different for different types of fog and clouds. The classification of clouds and fog is highly related to the amount of LWC and its origin. The combination of LWC and its origin allows to readily predict the types of conditions that will be, most likely, in the vicinity of the FSO links [19]. Fog that has very low densities contains very small amount of water and so eventually results in lower values of LWC i.e., about $0.05 \mathrm{~g} / \mathrm{m}^{3}$ for a moderate fog (visibility range around $300 \mathrm{~m}$ ). Much higher values of LWC (around $0.5 \mathrm{~g} / \mathrm{m}^{3}$ ) result in the formation of thick or dense fog (visibility range of about $50 \mathrm{~m}$ ) [10]. Fog characterized by several physical parameters like particle size distribution, temperature, humidity and LWC has been extensively modeled by drop size distribution and visibility range.

The analysis of the measured LWC is provided in Fig. 5.

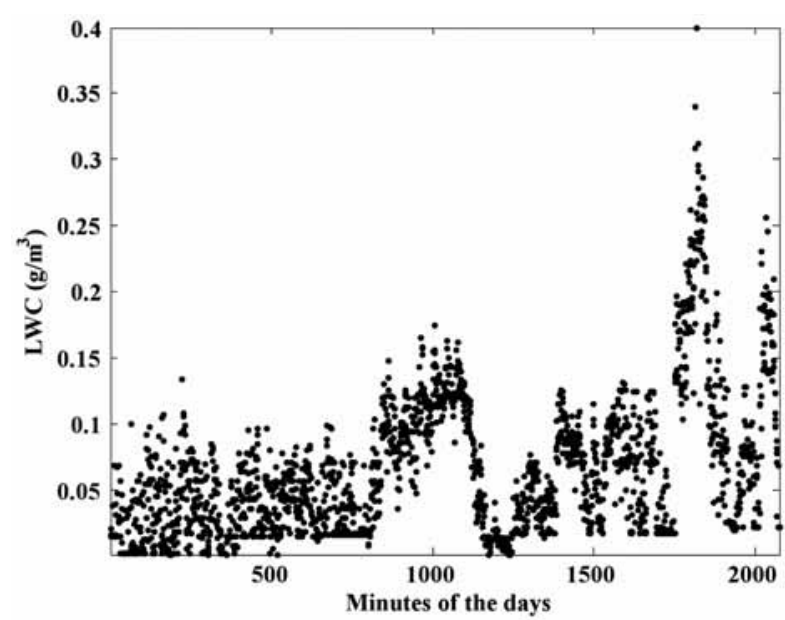

Fig. 5 Analysis of measured LWC

The horizontal axis of Fig. 5 shows the minutes of the days and the vertical axis shows the LWC measured in $\mathrm{g} / \mathrm{m}^{3}$. The descriptive statistics of the measured LWC is provided in Table 3.
Descriptive statistics of the measured LWC

Tab. 3

\begin{tabular}{|c|c|c|}
\hline Serial No & Statistical Quantity & Values \\
\hline 1 & Sample Size & 2073 \\
\hline 2 & Range & 0.399 \\
\hline 3 & Mean & 0.06422 \\
\hline 4 & Variance & 0.00281 \\
\hline 5 & Std. Deviation & 0.05304 \\
\hline 6 & Std. Error & 0.00116 \\
\hline 7 & Skewness & 1.4467 \\
\hline 8 & Min & 0.001 \\
\hline 9 & $25 \%(Q 1)$ & 0.021 \\
\hline 10 & $50 \%($ Median $)$ & 0.053 \\
\hline 11 & $75 \%(Q 3)$ & 0.093 \\
\hline 12 & $90 \%$ & 0.128 \\
\hline 13 & $95 \%$ & 0.17 \\
\hline 14 & Max & 0.4 \\
\hline
\end{tabular}

It is obvious from Table 3 that $95 \%$ of the time the LWC remains below $0.17 \mathrm{~g} / \mathrm{m}^{3}$.

We applied curve fitting techniques to find the best fit distribution model for measured LWC. We compared all the continuous distribution on the LWC data by analysing their PDF and CDF. We performed comparison among all the distribution by comparing the QQ plot. The PDF, CDF of the measured LWC data is provided in Figs. 6 and 7 respectively. Fig. 6 shows that both selected distribution models can be used for statistical characterization of LWC under fog conditions. But the results of QQ plot suggest that Gamma distribution is a better suited model for LWC than compared Beta distribution.

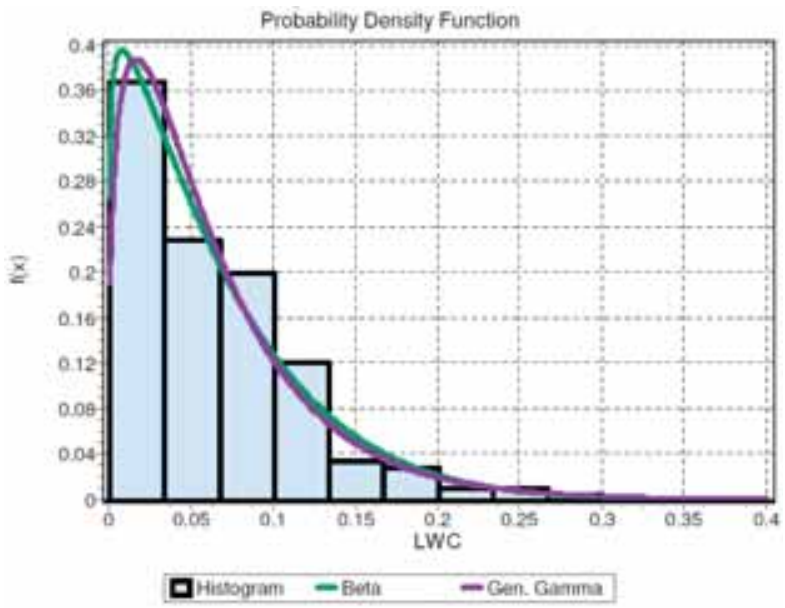

Fig. 6 PDF over the histogram of measured LWC 


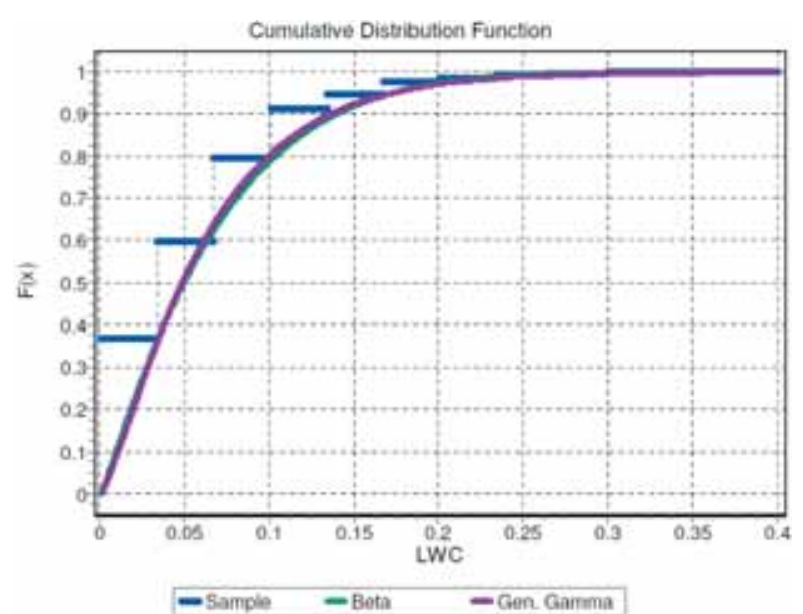

Fig. 7 CDF of the measured $L W C$

We performed the goodness of it by observing the QQ plot of the measured LWC. The QQ plot is provided in Fig. 8. It is obvious from Fig. 8 that Gamma distribution model is performing well as compared to Beta distribution. QQ plot for Gamma distribution is converging but QQ plot for Beta distribution is deviating for higher values of LWC. The distribution function for three parameters Gamma distribution is provided in equation (2)

$$
f(x)=\frac{(x-y)^{\alpha-1}}{\beta^{\alpha} \Gamma(a)} \exp (-(x-y) / \beta)
$$

Where $\alpha$ is a shape parameter $(\alpha>0), \beta$ is scale parameter $(\beta>0)$ and $\gamma$ is location parameter. We computed the optimum parameters for Gamma distribution for measured visibility which are provided in Table 2.

We concluded that Gamma distribution (3 parameters Gamma Distribution) is the best suited model for measured LWC in the absence of precipitation. The proposed distribution model can be used under fog conditions in the absence of precipitation.

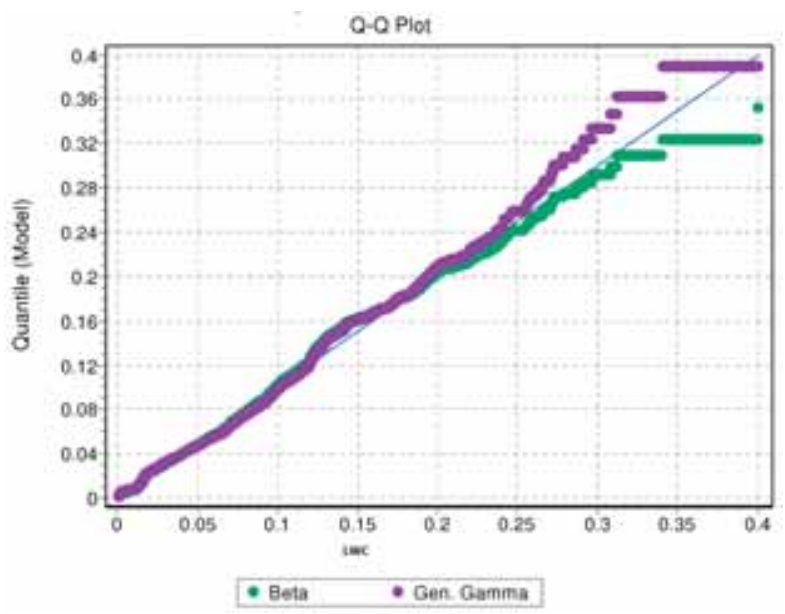

Fig. $8 Q Q$ Plot for measured data of $L W C$

\section{Conclusions}

A detailed statistical analysis of 5 months real time measured data of visibility and LWC for terrestrial FSO links has been presented to find the best fit distribution model for visibility and LWC. Our findings show that in reduced visibility, due to fog, 4 parameters Gamma distribution best fit the measured data of visibility and 3 parameters Gamma distribution is the best fit distribution model for LWC in the absence of precipitation. Both proposed model can be used under fog conditions ranging from dense fog to light fog and in the absence of precipitation.

\section{Acknowledgments}

The measurement campaign in Prague was supported by the Ministry of Education, Youth and Sports of the Czech Republic under the project No. OC09076 in the framework of COST IC0802. The prime author has a PhD studentship through the Higher Education Commission, Pakistan.

\section{References}

[1] AHARONOVICH, M., ARNON, S.: Performance Improvement of Optical Wireless Communication through FOG with a Decision Feedback Equalizer, J. of Optical Society of America, 2005, vol. 22, no. 8, p. 1646-1654.

[2] MUHAMMAD, S. S., FLECKER, B., LEITGEB, E., GEBHART, M.: Characterization of FOG Attenuation in Terrestrial Free Space Links, J. of Optical Engineering, 2007, vol. 46, no. 4, p. 066001.

[3] GRABNER, M., KVICERA, V.: The Wavelength Dependent Model of Extinction in FOG and Haze for Free Space Optical Communication, Optics Express, 2011, vol. 19, no. 4, p. 3376-3386.

[4] ZHEN-WEI, Z., ZHEN-SEN, W.: Millimeter-wave Attenuation Due to FOG and Clouds, Int'n J. of Infrared and Millimeter Waves, 2000, vol. 21, no 10, p. 1607-1615.

[5] TONNA, G., VALENTI, C.: Optical Attenuation Coefficients and Liquid Water Content Relationship in FOG, at Seventy Four Wavelengths from 0.35 to $90 \mu$ m", Atmospheric Environment, 1983, vol. 17. No. 10, p. 2075-2080.

[6] MUHAMMAD, S. S., AWAN, M. S., REHMAN, A.: PDF Estimation and Liquid Water Content Based Attenuation Modeling for FOG in Terrestrial FSO Links", Radioengineering, 2010, vol. 19, no. 2, p. 228-236. 
[7] KONTOGEORGAKIS, C.: Millimeter through Visible Frequency Waves through Aerosols Particle Modeling, Reflectivity and Attenuation, Master Thesis at Virginia Polytechnic Institute and State University, 1997.

[8] ROBERTS, R. E., SEEKAMP, L. N.: Infrared Attenuation by Aerosols in Limited Atmospheric Visibility: Relationship to Liquid Water Content”, IDA PAPER P-1394, March 1979.

[9] SHETTLE, E. P.: Models of Aerosols, Clouds, and Precipitation for Atmospheric Propagation Studies, AGARD Conference Proceedings, v. 454, n. 15, 1989.

[10] AWAN, M. S.: Statistical-Dynamical Channel Modeling of Outdoor Optical Wireless Links, Ph.D Thesis, TU Graz, Austria, 2010.

[11] Czech Hydrometeorological Institute, "http://www.chmi.cz/meteo/ok/klemintroe.html”, visited on 10 October 2010.

[12] GEBhART, M., LEITGEB, E., MUHAMMAD, S. S., FLECKER, B., CHLESTIL, C., Al NABOULSI, M., DE FORNEL, F., SIZUN, H.: Measurement of Light Attenuation in Dense FOG Conditions for Fso Applications. SPIE Proceedings, 5891, 2005.

[13] KOLKA, Z., WILFERT, O., KVICALA, R., FISER, O.: Complex Model of Terrestrial FSO Links. SPIE Proceedings, 6709:67091J.167091J.8, 2007.

[14] JACOBSON, M. Z.: Fundamentals of Atmospheric Modeling. Cambridge University Press, The Edinburgh Building, Cambridge CB2 2RU, UK, 2nd edition edition, 2005.

[15] KIM, I. I., KOREVAAR, E.: Availability of Free Space Optics (FSO) and Hybrid FSO/RF Systems. SPIE Proceedings, 4530:84-95, 2001.

[16] PIERCE, R. M., RAMAPRASAD, J., EISENBERG, E.: Optical Attenuation in Fog and Clouds. SPIE Proceedings, 4530:58-71, 2001.

[17] Kruse, P. W. et al.: Elements of Infrared Technology: Generation, transmission and detection. J. Wiley and sons, New York, 1962.

[18] AL NABOULSI, M., SIZUN, H., FORNEL, F. D.: Fog Attenuation Prediction for Optical and Infrared Waves. SPIE Proceedings, $43(2): 319-329,2004$.

[19] MUHAMMAD, S. S., AWAN M. S., REHMAN, A.: PDF Estimation and Liquid Water Content Based Attenuation Modeling for Fog in Terrestrial FSO links, J. of Radioengineering, v. 19, 2010. 